\title{
Indicações para fins terapêuticos da toxina botulínica do tipo A no uso odontológico: uma revisão de literatura
}

\author{
Indications for therapeutic purposes of botulinum toxin type $A$ in dental use: a review of \\ literature
}

Indicaciones para fines terapéuticos de la toxina botulínica tipo A en uso dental: una revisa de literatura

Alan Alves da Silva ${ }^{1 *}$, Dalmario Gaia Nepomuceno Filho ${ }^{1}$ Lucas Magalhães Bomfim¹, Lívia Adélia Mendes Silva ${ }^{1}$, Mirella Soares da Cunha ${ }^{1}$, Savana Costa de Albuquerque ${ }^{1}$, Weizia Gomes da Rocha $^{1}$, Monaly de Oliveira Lima², Ana Fernanda Cumarú Duque ${ }^{3}$, João Francisco Tenório Neto'.

\section{RESUMO}

Objetivos: Esse estudo tem como foco efetuar uma revisão de literatura a respeito das indicações para fins terapêuticos da utilização da toxina botulínica do tipo A no uso odontológico para o tratamento de pacientes que apresentam bruxismo e sorriso gengival em excesso. Métodos: Foi efetivada uma revisão de literatura, tendo como forma de abordagem aspecto descritivo com caráter informativo, em que o processo de construção ocorreu através de pesquisas presentes em acervos científicos, apresentando como descritores: A utilização da toxina botulínica como fins terapêuticos, utilização no bruxismo e toxina botulínica tipo A para correções gengivais. Resultados: Visando amenizar os problemas relacionados ao bruxismo e ao sorriso gengival devolvendo ao paciente não só fins estéticos, como terapêuticos, destaca-se a utilização da toxina botulínica, por se tratar de um procedimento não invisível e de resultado imediato. Conclusão: A toxina botulínica do tipo A, é uma escolha de primeiro instante ao que se trata de procedimentos terapêuticos, possui um resultado satisfatório em pouco tempo, devido o controle da hiperatividade da musculatura do masseter e do temporal, sendo de fácil manuseio pelos Cirurgiões-dentistas qualificados.

Palavras-chave: Estética, Toxinas botulínicas tipo A, Sorriso gengival, Uso terapêutico.

\begin{abstract}
Objectives: This study focuses on performing a literature review regarding the indications for therapeutic purposes of the use of botulinum toxin type $A$ in dental use for the treatment of patients with brucism and excessive gingival smile. Methods: A literature review was carried out, using as a descriptive aspect with an informative character, in which the construction process occurred through research in scientific collections, presenting as descriptors: The use of botulinum toxin as therapeutic purposes, use in bruxism and botulinum toxin type A for gingival corrections. Results: In order to alleviate the problems related to bruxism and gingival smile, returning to the patient not only aesthetic but also therapeutic purposes, the use of botulinum toxin stands out, as it is a non-invisible procedure with immediate results. Conclusion: Botulinum toxin type $A$ is a first choice when it comes to therapeutic procedures, has a satisfactory result in a short time, due to the control of the masseter and temporal muscle hyperactivity, being easy to handle by qualified dental surgeons.
\end{abstract}

Key words: Esthetics, Botulinum toxins type A, Gingival smile, Therapeutic use.

\section{RESUMEN}

Objetivos: Este estudio se centra en realizar una revisión de la literatura sobre las indicaciones con fines terapéuticos del uso de la toxina botulínica tipo $\mathrm{A}$ en el uso dental para el tratamiento de pacientes con bruxismo y sonrisa gingival excesiva. Métodos: Se realizó una revisión de la literatura, utilizando como

${ }^{1}$ Centro Universitário CESMAC (CESMAC), Maceió - AL. *E-mail: alanalves250odonto@hotmail.com ¿2Universidade Federal de Alagoas (UFAL), Maceió - AL.

${ }^{3}$ Centro Universitário Tabosa de Almeida (UNITA), Caruaru - PE.

SUBMETIDO EM: 6/2020

ACEITO EM: 7/2020

PUBLICADO EM: 9/2020 
aspecto descriptivo con un carácter informativo, en el que el proceso de construcción se produjo a través de la investigación en colecciones científicas, presentando como descriptores: el uso de la toxina botulínica como fines terapéuticos, el uso en bruxismo y toxina botulínica tipo A para correcciones gingivales. Resultados: Para aliviar los problemas relacionados con el bruxismo y la sonrisa gingival, volviendo al paciente no solo con fines estéticos sino también terapéuticos, destaca el uso de la toxina botulínica, ya que es un procedimiento no invisible con resultados inmediatos. Conclusión: La toxina botulínica tipo A, es una primera opción cuando se trata de procedimientos terapéuticos, tiene un resultado satisfactorio en poco tiempo, debido al control de la hiperactividad de los músculos maseteros y temporales, y es fácil de manejar por cirujanos dentales calificados.

Palabras clave: Estética, Toxinas botulínicas tipo A, Sonrisa gingival, Uso terapêutico.

\section{INTRODUÇÃO}

Os primeiros estudos que envolviam a utilização da toxina botulínica foram feitos na década de 70 pelo pesquisador Scott e sua equipe, possuía como principal objetivo analisar o comportamento dela para fins terapêuticos. O estudo se estabeleceu através da análise das ações referentes ao comportamento da musculatura extraocular de primatas antropoides nas reparações de correções do distúrbio que afeta o paralelismo entre os olhos. Em meados à década de 90 passou a ser não só utilizada no estrabismo em seres humanos, assim como em determinados transtornos referentes a área neuromuscular relacionados aos distúrbios nas articulações temporomandibular (ATM) (SEVILHA FM, et al., 2011).

A toxina botulínica (TXB) é obtida através da síntese realizada pela bactéria de origem anaeróbica chamada Clostridium botulinum dos quais podem produzir sete tipos de toxina botulínica, do qual a do tipo A apresenta-se como sendo a mais eficiente na aplicação do uso odontológico. A toxina botulínica utilizada é estabelecida por processos industrializados dos quais purificam, deixando-a apta a ser usada em doses adequadas que não prejudicam à saúde (OLIVEIRA MT e MOLINA RO, 2011), atualmente ela é bastante procurada pelos profissionais capacitados, devido seu potencial benéfico de diminuir o bruxismo, disfunção têmporomandibular, processos relacionados a hipertrofia do masseter e exposição gengival acentuada, isso ocorre porque ela age de forma bloqueadora não disponibilizando o neurotransmissor da acetilcolina e impedindo por sua vez a constrições musculares (PEDRON IG, 2014).

A princípio, a TXB era manuseada apenas por profissionais da medicina, dos quais possuíam fins terapêuticos. Entretanto, após várias análises e resultados de pesquisas, passou a ser manipulada também por Cirurgiões-dentistas (CD) não apenas em procedimentos terapêuticos como também na harmonização, demostrando-se ser bastante eficaz (MATOS MB, et al., 2017).

Diante da elevada conjuntura e grande importância da aplicação da toxina botulínica, este artigo tem como objetivo executar uma revisão da literatura a respeito das escolhas terapêuticas referente a utilização da toxina botulínica tipo A pelos Cirurgiões-Dentistas, referente ao tratamento da correção do sorriso gengival e do bruxismo, mostrando dessa forma técnica menos invasiva, sobretudo em pacientes com aumento da atividade funcional dos músculos que envolvem o sorriso, bem como em outras decorrências que atinge a musculatura responsáveis pelas disfunções temporomandibulares e a dor miofacial.

\section{MÉTODOS}

Em primeiro instante, foi adquirido a execução de 28 artigos referentes a toxina botulínica do tipo A com fins terapêuticos. Dos quais, 14 estavam repetidos nas referidas bases de dados, ficando 14 artigos para a leitura dos títulos, dentre eles, 4 foram excluídos por não estarem correspondo as principais adaptações dos critérios que estavam sendo focados.

Os 10 artigos designados para observação dos resumos, dos quais 3 foram eliminados, ficando apenas 7 para assegurar esse estudo. Após a revisão dos artigos nenhum outro trabalho foi incorporado. Ao fim das investigações dos dados, e o fluxograma da Figura 1 mostra de forma clara todas as formas de busca pelas pesquisas elegidas para a confecção desta revisão. 
Figura 1 - Fluxograma dos estudos identificados nas bases de dados.

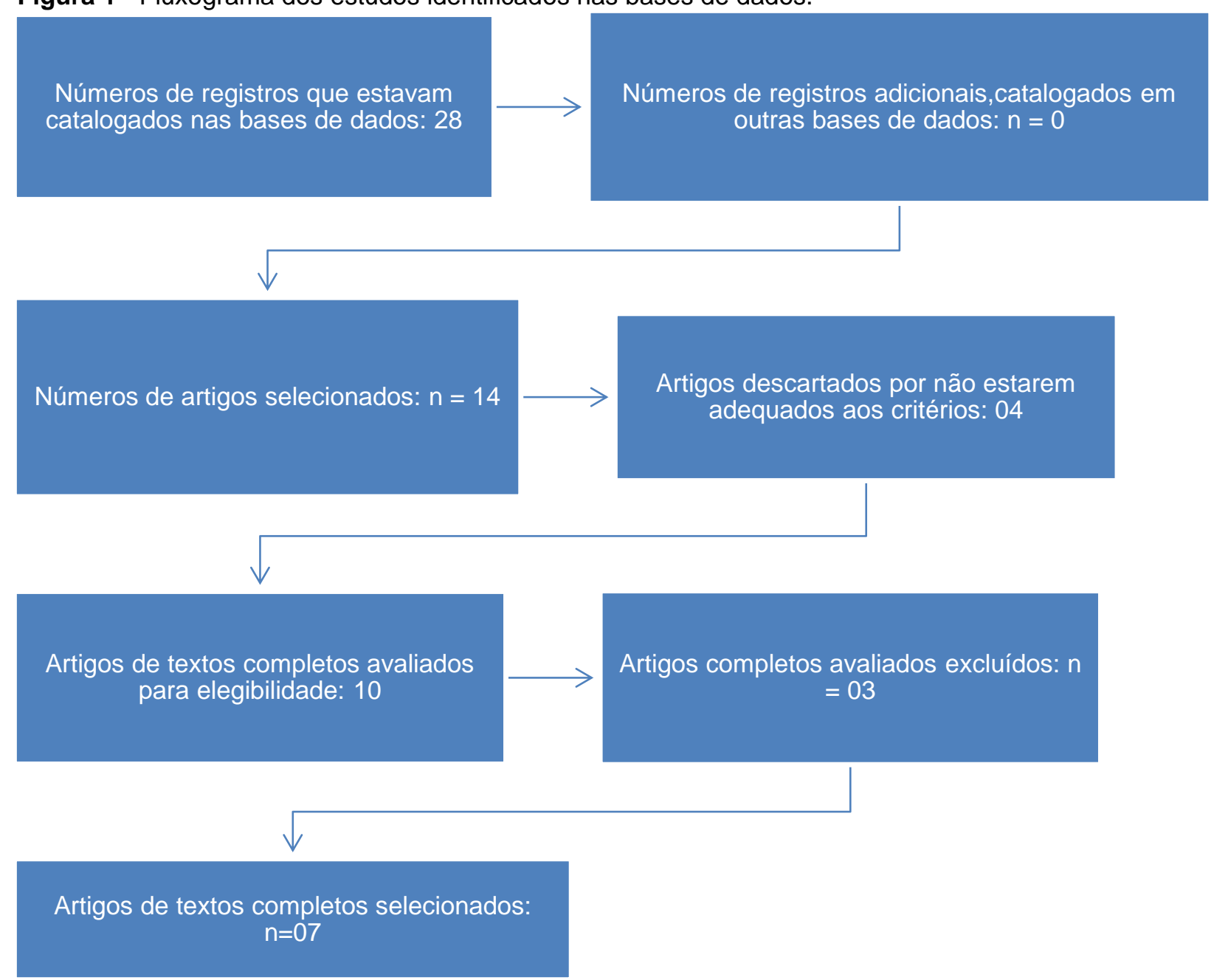

Fonte: Silva AA, et al., 2020.

Para a elaboração deste trabalho foi executada uma revisão de literatura, sendo realizada entre o período de dezembro de 2019 a abril de 2020, dos quais foram examinados artigos divulgados em bases de dados eletrônicos Literatura Latino Americana e do Caribe em Ciências da Saúde (LILACS), Literatura Internacional em Ciência da Saúde (MEDLINE) e Scientific Electronic Library Online (SCIELO) e livros que estavam disponíveis nos acervos bibliográficos presentes na biblioteca central do Centro Universitário de Maceió (CESMAC).

Foram consultados textos em língua portuguesa e inglesa, em que referia-se a aplicação da toxina botulínica e seus benefícios, possuindo como forma de restrições os períodos aos quais os artigos foram publicados, os que não estavam presentes na íntegra, relatos de casos, séries de casos, ensaios não controlados e os artigos que não dispunham expressividade com a temática estabelecida, sendo eleitos os textos científicos que exibiam e constavam o real objetivo do trabalho, analisando a relevância da utilização da toxina botulínica (TXB) tipo A na área odontológica certificando o melhor procedimento para a aquisição de seguimentos aceitáveis e satisfatórios para o paciente, sendo selecionadas publicações científicas no período entre 2014 e 2020, com ênfase no período entre 2015 e 2020, dispondo dos seguintes descritores: utilização da Toxina botulínica tipo A, Bruxismo e Sorriso gengival.

\section{RESULTADOS E DISCUSSÃO}

Os artigos possuíram suas informações concentradas nos tópicos: autor/ano, país, objetivo e resultados, os quais foram tabulados e apresentados no Quadro 1. 
Revista Eletrônica Acervo Saúde / Electronic Journal Collection Health | ISSN 2178-2091

Quadro 1 - Delineamento, métodos e principais desfechos dos estudos selecionados.

\begin{tabular}{|c|c|c|c|c|}
\hline Autor & Ano & País & Objetivo & Resultados \\
\hline MATOS MB, et al. & 2017 & Brasil & $\begin{array}{c}\text { Fazer uma revisão literária sobre o uso da } \\
\text { toxina botulínica na correção } \\
\text { do sorriso gengival e descrever } \\
\text { seu protocolo de uso (AU) }\end{array}$ & $\begin{array}{c}\text { O uso desta substância na odontologia, pode ser mais } \\
\text { uma opção para amenizar ou corrigir o sorriso gengival } \\
\text { por hiperfunção muscular ou associar a outros tipos } \\
\text { de tratamentos. }\end{array}$ \\
\hline CALZA SC. et al. & 2015 & Brasil & $\begin{array}{c}\text { O sorriso é a mais bela das expressões } \\
\text { faciais e está diretamente relacionado com } \\
\text { bem-estar, felicidade, prazer entre outros. } \\
\text { Fisiologicamente é o resultado da exposição } \\
\text { dos dentes e da gengiva durante a contração } \\
\text { de um grupo muscular. }\end{array}$ & $\begin{array}{c}\text { Foi tratada com } 10 U \text { bilaterais de toxina botulínica tipo } \\
\text { A que resultou em um sorriso harmônico e } \\
\text { esteticamente mais agradável. }\end{array}$ \\
\hline $\begin{array}{l}\text { OLIVEIRA MT e } \\
\text { MOLINA RO. }\end{array}$ & 2011 & Brasil & $\begin{array}{c}\text { Elucidar a aplicação do Botox como } \\
\text { alternativas terapêuticas para tratamento do } \\
\text { sorriso gengival. }\end{array}$ & $\begin{array}{c}\text { O Botox pode ser utilizado como alternativas } \\
\text { terapêuticas para o tratamento do sorriso gengival } \\
\text { somente quando a etiologia for causada por hiper } \\
\text { contração labial. }\end{array}$ \\
\hline DUTRA MB, et al. & 2011 & Brasil & $\begin{array}{c}\text { Propôs a avaliar a influência da quantidade } \\
\text { de exposição gengival na estética do sorriso } \\
\text { para os sexos feminino e masculino, e se } \\
\text { existe diferença de opinião entre } \\
\text { ortodontistas, clínicos gerais e pessoas } \\
\text { leigas. }\end{array}$ & $\begin{array}{l}\text { O sorriso mais estético para o indivíduo do sexo } \\
\text { feminino, tanto para ortodontistas, clínicos gerais e } \\
\text { leigos, foi aquele em que o lábio superior repousa na } \\
\text { margem cervical dos incisivos superiores, mostrando } \\
\text { toda a coroa dos incisivos ( } p \leq 0,05 \text { ). Para o indivíduo } \\
\text { do sexo masculino, o sorriso mais estético, para } \\
\text { pessoas leigas, foi com o lábio na altura da margem } \\
\text { cervical dos incisivos superiores ( } p \leq 0,05) \text {, sendo que } \\
\text { ortodontistas e clínicos gerais consideraram tanto o } \\
\text { lábio na altura da margem cervical como o lábio } \\
\text { superior cobrindo os incisivos superiores em } 2 \mathrm{~mm} \\
\text { como os mais estéticos }(p \leq 0,05) \text {. }\end{array}$ \\
\hline
\end{tabular}

REAS/EJCH | Vol.Sup.n.58 | e4348 | DOI: https://doi.org/10.25248/reas.e4348.2020 Página 4 de 10 
Revista Eletrônica Acervo Saúde / Electronic Journal Collection Health | ISSN 2178-2091

\begin{tabular}{|c|c|c|c|c|}
\hline Autor & Ano & País & Objetivo & Resultados \\
\hline $\begin{array}{l}\text { KEANEY TC e } \\
\text { ALSTER TS. }\end{array}$ & 2016 & EUA & $\begin{array}{l}\text { Fornecer uma revisão das características } \\
\text { anatômicas masculinas pertinentes e } \\
\text { informações clínicas atualizadas sobre o uso } \\
\text { da toxina botulínica em homens. }\end{array}$ & $\begin{array}{c}\text { Apenas dois estudos foram responsáveis pelo sexo no } \\
\text { desenho do estudo ou na análise de } \\
\text { subgrupos. Ambos os estudos descobriram que a } \\
\text { abobotulinumtoxinA é menos eficaz em homens. Um } \\
\text { estudo adicional que examinou a dose de } \\
\text { onabotulinumtoxinA em homens constatou que doses } \\
\text { mais altas do que o normalmente usado em mulheres } \\
\text { eram mais eficazes. Não houve mais eventos adversos } \\
\text { em participantes do sexo masculino em nenhum } \\
\text { estudo. }\end{array}$ \\
\hline $\begin{array}{l}\text { MACEDO ACVB, } \\
\text { et al. }\end{array}$ & 2012 & Brasil & $\begin{array}{c}\text { Revisar literatura atual acerca dos fatores } \\
\text { etiológicos do sorriso gengival (hiperplasia } \\
\text { gengival, extrusão dento-Alveolar anterior, } \\
\text { excesso vertical de maxila (VME), e lábios } \\
\text { superiores curtos ou hiperativos durante o } \\
\text { sorriso, erupção passiva alterada) assim } \\
\text { como da simiotécnica relacionada, visando } \\
\text { auxiliar o cirurgião-dentista na tomada de } \\
\text { decisão e no estabelecimento do plano de } \\
\text { tratamento adequado para cada uma dessas } \\
\text { etiologias. }\end{array}$ & $\begin{array}{l}\text { A etiologia do sorriso gengival é variada e sua correta } \\
\text { identificação é fundamental para confecção de um } \\
\text { plano de tratamento eficaz. Para podermos } \\
\text { diagnosticar precisamente o fator causador } \\
\text { preponderante em cada caso, alguns aspectos clínicos } \\
\text { devem ser avaliados. }\end{array}$ \\
\hline MAGRO AKD. & 2015 & Brasil & $\begin{array}{l}\text { O sorriso é a mais bela das expressões } \\
\text { faciais e está diretamente relacionado com } \\
\text { bem-estar, felicidade, prazer entre outros. }\end{array}$ & $\begin{array}{l}\text { Foi tratada com } 10 U \text { bilaterais de toxina botulínica tipo } \\
\text { A que resultou em um sorriso harmônico e } \\
\text { esteticamente mais agradável. Considerações finais: o } \\
\text { sorriso gengival é considerado uma desordem estética } \\
\text { por muitos pacientes e pode ser tratado por meio da } \\
\text { utilização da toxina botulínica. }\end{array}$ \\
\hline
\end{tabular}

Fonte: Silva AA, et al., 2020. 


\section{Histórico}

Durante o século XVIII, na Europa, o agente causador de uma doença relacionado ao botulismo foi reconhecido pelo pesquisador Van Ermengem, ao qual denominou Bacillus botulinus. No ano de 1897 Kempner, posteriormente, comprovou que o experimento realizado através de cultura feito por Van Ermengem gerava uma substância da qual se fosse aplicada inativamente controlava à síntese de uma específica antitoxina presente no sangue de cabras.

Esse experimento foi o primeiro indicativo científico do qual demostrou que uma antitoxina seria capaz de paralizar a toxina botulínica neutralizando desta forma sua toxidade, a doença referente ao botulismo ou até mesmo a morte do indivíduo. No ano de 1922 este agente etiológico foi denominado como sendo C. botulinum (MATOS MB, et al., 2017)

No ano de 1989, a aplicação da TXB foi concedida pela Food and DrugAdministration (FDA) do qual sua utilização era aconselhada para indivíduos que tinham distúrbio que afetavam o paralelismo entre os olhos conhecido como estrabismo e espasmos musculares (blefaroespasmo). No Brasil a aprovação da aplicação de toxina botulínica ocorreu no ano de 2000 pela Agência Nacional de Vigilância Sanitária (ANVISA).

Em 2011, os CD's foram conceituados e licenciados pelo Conselho Federal de Odontologia (CFO) a utilizar toxina botulínica em procedimentos que possuíam fins terapêuticos e estéticos, limitando-se a sua área de atuação, possuindo como agentes delimitadores a parte lateral da face entre a estrutura do tragus e na região frontal entre o início do cabelo até a estrutura do osso hióideo. Na atualidade quatro marcas comerciais são mais conhecidas na utilização dos profissionais, sendo elas: Botox ${ }^{\circledR}$; DysportTM $\AA ;$ Xeomin $\AA$ e Prosigne® (OKAJIMA M, 2013).

\section{Mecanismo de ação}

A toxina botulínica é um agente biológico formado naturalmente pelos agentes bacterianos anaeróbicos gram positivos, conhecido como Clostridium botulinum, dos quais quando entram em contato com os músculos da face, passam a ter comunicação direta com as terminações nervosas, causando a imobilização dos canais onde possuem cálcio, inibindo a passagem da acetilcolina e por resultado desse efeito biológico promove o repouso ou paralisação do músculo alvo de forma passageira (MOCK D, 2015).

O processo de recomposição fisiológica de modo normal acontece gradativamente, no período estimado entre dois a três meses depois de sua aplicação. Desta forma, percebe-se que a restituição da estagnação decorrente da paralisação dos músculos decorre por meio de mecanismos sendo eles: o brotamento neural, onde surgi novos brotos axonais e a reinervação da região onde ocorre a formação de novas placas de terminações menores.

O músculo onde passou pelo procedimento passa por regeneração por conta das proteínas presentes nas vesículas de acetilcolina do qual comumente é reconstituída entre um e quatro meses (CALZA SC, et al., 2015).

Após a aplicação da TXB do tipo A, seu resultado começa a ser iniciado depois das 24 a 48 horas após os procedimentos injetáveis. Referente aos aspectos clínicos é observado paralisia expressiva após as primeiras 48 horas podendo chegar até os 10 dias, sendo que o resultado mais expressivo acontece na segunda semana pós o procedimento ser realizado.

Novos elementos interligados SNARE (soluble NSF attachment receptor) começam a se formar depois de 2 a 3 meses da aplicação, dado que neste espaço de tempo as cadeias mais leves da toxina encontramse inertes e as extremidades nervosas restauram sua funcionalidade. Deste modo, a efetividade da aplicação injetável da TXB do tipo A permanecem em média 3 meses (KANE M e SATTLER G, 2016).

A localidade respectiva da aplicação deve ser estudada e estabelecida pelo CD, pois o local estabelecido e a concentração da sua dosagem são diretamente correlacionadas com o tempo médio da durabilidade e eficácia da TXB tipo A. Sua atuação em potencial máximo é expressiva entre a primeira semana podendo chegar até os 14 dias sendo sua duração estabelecida até seis meses, dependendo de qual produto e a técnica que irá ser utilizada (SPOSITO MMM e TEIXEIRA SAF, 2014). 


\section{Indicações e Contraindicações}

No uso odontológico a toxina botulínica é mais utilizada para casos em que o paciente sofre com dores provocadas pela disfunção temporomandibular, pela Síndrome de Meige conhecida como distonia orofacial, pacientes que apresentam hábitos parafuncionais principalmente o bruxismo, atrofiamento da musculatura do masseter, ptialismo ou polissialia, desarmonizarão da assimetria labial, dores na face e apresentação acentuada da gengiva (PANOSSIAN AJ e BLOCK MS, 2010).

A TXB demostrou-se eficiente para o tratamento de inúmeras situações estomatológicas e, nos dias atuais sua utilização tem sido adequada para pessoas que possuem hiperfunção das musculatura que circundam o sorriso gengival bastante destacado, além de ser de suma importância para o controle das disfunções temporomandibulares (SENISE IR, et al., 2015).

Em casos que são levados em considerações as contraindicações da utilização da toxina botulínica podese destacar: na gravidez, lactação, quando o paciente apresenta uma grande sensibilidade de forma particularizada a toxina botulínica, lactose e albumina, doenças sistêmicas referentes a musculatura e neurodegenerativas da qual é uma doença causada por uma falha de comunicação entre os nervos e os músculos, e na utilização continuo de antibiótico aminoglicosídico dos quais são medicamentos da classe bactericidas, que são inibidores de síntese proteica das bactérias sensíveis a eles onde podem potencializar a ação da toxina (KLEIN AW, 2002).

Outras contraindicações que podem serem relacionadas com a aplicação da TXB do tipo A são integrais de forma absoluta quando o indivíduo demonstra princípios alérgicos a droga utilizada, ou em outros casos quando o paciente possui infecções ou até mesmo processos inflamatórios no local que irá ocorrer o bloqueio bioquímico, e/ou relacionadas a pacientes que apresentam um quadro de enfermidade neuromusculares relacionadas a contrações musculares involuntárias, súbitas, anormais, apresentam distúrbios da coagulação sanguínea, recusa ou até mesmo à falta de cooperação do paciente em relação a técnica utilizada pelo profissional (PEDRON IG, 2014).

\section{Vantagens e desvantagens}

Quando é utilizado a da toxina botulínica do tipo A o paciente que sofre com bruxismo nota imediatamente o conforto novamente diminuindo em instantes a dor que estava sendo acometido, esse fator se dar por causa do relaxamento dos tecidos envolvidos, dos quais amenizam o estresse e fadiga das musculaturas na região da aplicação. Esse conforto pode-se perdurar até 6 meses, e logo após esse tempo o músculo volta a sua função normal podendo fazer com quer o paciente sinta as dores novamente (E SILVA NETO JMDEA, et al., 2019).

Devido a toxina botulínica tipo A agir na musculatura periférica ela beneficia pacientes que apresentam casos graves de bruxismo, sendo importante ressaltar que a mesma não prejudica o sistema nervoso central por não agir nessa parte, expondo assim como sendo benéfica e vantajosa na diminuição dos sintomas dolorosos (PEDRON IG, 2014).

Um dos grandes benefícios da utilização da toxina é que se trata de um procedimento não tão invasivo podendo ser em quase todos os casos reversível. Desta forma, se porventura ocorrer determinada insatisfação tanto pelo profissional quanto pelo paciente, o quadro conseguirá ser retrocedido.

Observa-se nas práticas clínicas que a utilização da toxina é uma forma bastante benéfica para fins terapêuticos quando é levado em consideração outros procedimentos que necessitam de cirurgias, esse fato deve-se por causa da técnica ser extremamente conservadora, tendo efeito rápido, seguro e benéfico (MAGRO AKD, 2015).

Quando ocorre procedimentos irregulares prejudicando a harmonização no caso de assimetrias, queda da pálpebra superior (ptoses) e problemas relacionados com a expressão facial em decorrência do repouso excessivo dos músculos, os recursos terapêuticos apropriados são incitação muscular através de fisioterapia e procedimentos de laserterapia (SPOSITO MMM, 2004). 
Estudos feitos por Senise IR, et al. (2015) a toxina botulínica é uma técnica da qual fornece como principal vantagem a forma simplória de se efetuar a aplicação, desde que seja manipulada por um profissional qualificado, além de possuir grande conformidade e ser compatível com o organismo, existe poucos relatos de complicações pós sua aplicação, além de seu efeito ser rápido. Quando o profissional apresenta um manuseio no ato da aplicação bem feita, os possíveis efeitos colaterais são quase nulos. Do contrário, pode ser visto como desvantagens pequenos incômodos e ligeira contusão na área da aplicação.

Possíveis efeitos colaterais que podem ser vistos depois do procedimento terapêutico feito com a toxina botulínica são: sangramento no tecido subcutâneo, possíveis hematomas, dor, edema se não possuir cuidados prescritos pelo $C D$, podendo ocasionar até possíveis infecções locais, além de ocasionar fraqueza muscular nos locais do tratamento. Em alguns casos pode acontecer do paciente se queixar de cefaleias e raramente alergia. Um dos efeitos que pode ser acometido é a perda funcional devido ao relaxamento do muscular (MATOS MB, et al., 2017).

A utilização de doses incorretas na aplicação da TXB pode ser provocada por técnicas incorretas pelo manejador durante sua aplicação. Deve-se levar em consideração as condições de armazenamento, pois se não estiverem armazenados em locais corretos em temperaturas ideias podem diretamente influenciar na sua eficácia. Das situações mais corriqueiras ocasionadas pela aplicação da toxina pode-se destacar a assimetria, e comumente não se evidencia quando o paciente está em repouso, mas sim quando o mesmo encontra-se em função ativa, ou melhor, ao ato de sorrir. A diminuição mais intensa do músculo provoca uma pequena descida da parte mediana do lábio superior afetando diretamente a estética, além de provocar o comprometimento da mastigação do paciente (CALZA SC,et al., 2015).

\section{Indicações terapêuticas da toxina botulínica tipo A na Odontologia}

\section{Uso no bruxismo}

Esse estabelecimento se dar devido o processo de apertamento dos dentes, ocorrendo de forma involuntária principalmente quando o paciente está dormindo. Essa condição clínica é muito comum, podendo ser vista através do desgaste dos dentes, e acarretar até possíveis trincas dos elementos dentais. Outros casos como barulhos oclusais audíveis de ranger, tônus expandido, enxaqueca e dores na ATM (dores e descomodidades, problemas mastigatórios, desarticulação, subluxação não traumáticas, barulhos articulares uni e bilateral, podendo possuir insuficiência de abertura normal da boca, além de desvio na abertura. Por ser bastante relaxante para essas enfermidades, a TXB proporcionará o repouso dos músculos envolvidos no processo mastigatório, diminuindo ou até mesmo bloqueando a dor e possibilitando uma funcionalidade da mandíbula conveniente (MACEDO ACVB, et al., 2012).

Quando se é feito a análise para a obtenção do diagnóstico de bruxismo no paciente é levado em consideração o desgaste dos elementos dentais, do histórico de hábitos, é observado possíveis mobilidades dentárias, se há fadiga e dores musculares pelos músculos responsáveis pela mastigação, incômodos na ATM. No entanto, ao ser feito o exame clínico a deterioração dos elementos dentais é o sinal mais se expressa o bruxismo (SENISE IR, et al., 2015).

Em estudos feitos foi observado que os pacientes que apresentavam bruxismo e foram tratados com a toxina botulínica através de aplicações injetáveis nos músculos masseter e temporal, demonstraram um resultado positivo ao que se refere ao equilíbrio na elevação da mandíbula, diminuindo desta forma os sintomas em torno de $70 \%$ dos casos. Porém para poder ser efetuado o planejamento da toxina botulínica nos músculos responsáveis pela mastigação, deve-se levar em consideração o equilíbrio, a menos que o indivíduo tenha uma discrepância significativa entre os dois lados da musculatura mandibular (KANE M e SATTLER G, 2016).

Em caso de pacientes com bruxismo, sempre a aplicação da TXB será nos músculos masseter e temporal, sendo utilizado uma conformidade e padronização da quantidade limitante de toxina injetada, sabendo-se a mesma quantidade que for aplicada no musculo masseter terá que ser a mesma proporção no temporal, obtendo-se dessa forma um equilíbrio entre o fechamento e abertura da boca evitando a fadiga e diminuindo os hábitos de apertamento dos elementos dentais (KANE M e SATTLER G, 2016). 


\section{Uso no sorriso gengival}

$\mathrm{Na}$ atualidade existem inúmeras formas de tratamentos para à correção do sorriso gengival. No entanto, determinados procedimentos por serem feitos de forma invasiva, faz com que o indivíduo tenha um tipo de demanda, certo medo, fazendo com que muitos desistam do tratamento proposto pelo CD (E SILVA NETO JMDEA, et al., 2019). Das técnicas utilizadas os protocolos sugeridos para essa tipo de correção são feitos através do reposicionamento da musculatura que envolve os lábios, utilizados nestes casos a cirurgia que é uma forma invasiva ou o preenchimento com a toxina botulínica, do qual faz com quer ocorra o relaxamento da musculatura responsáveis pelo lábio superior (MATOS MB, et al., 2017).

O sorriso do paciente pode ser julgado como gengival, quando existe o destacamento exagerado da gengiva, sendo considerada desta forma quando ela é superior à $3 \mathrm{~mm}$, podendo ser observado através de dados que quando a gengiva apresenta de 1 até $3 \mathrm{~mm}$ de descobrimento gengival considera-se como um padrão regular. Quando se é levado em consideração a harmonização facial do sorriso gengival a utilização da toxina botulínica é considerado como uma ótima metodologia a ser aplicada, pois com suas propriedades ativas ela faz com quer ocorra a redução da hiperatividade dos músculos que provocam a grande exposição gengival em excesso (FLÁVIO A, 2019).

Quando existe o problema da hiperfunção muscular do lábio fazendo com que exista a grande exposição gengival, a utilização da toxina botulínica é o procedimento a ser eleitos como de primeira opção, pois todos os músculos que estão na região orbicular da boca apresenta ativação quando se é realizado o sorriso, deste modo, quando se é aplicado a TXB, verifica-se a diminuição dos processos responsáveis pela constrição destas musculaturas que asseguram a elevação dos lábios, dessa maneira restringirá a exposição exagerada dos tecidos que formam a gengiva, transformando por sua vez o sorriso do paciente bem mais harmônico (PEDRON IG, 2014).

\section{CONSIDERAÇÕES FINAIS}

A utilização da TXB vem progressivamente sendo utilizada nos consultórios odontológicos devido sua técnica ser rápida, segura e reversível e acima de tudo minimamente invasiva, porém para o profissional executar tais técnicas é necessário está apto para desempenhar legalmente esses procedimentos, quando se é utilizada no tratamento terapêutico referente ao bruxismo observa-se significativamente a diminuição da sintomatologia dolorosa, tendo um resultado satisfatório em pouco tempo devido o controle da hiperatividade da musculatura do masseter e do temporal, já quando se é aplicada para correções do sorriso gengival a toxina apresenta um equilíbrio da força muscular, estabelecendo por sua vez um sorriso mais harmônico.

\section{REFERÊNCIAS}

1. CALZA SC, et al. Tratamento do sorriso gengival com toxina botulínica tipo A: relato de caso. RFO UPF, 2015; 20(10): 81-87.

2. DUTRA MB, et al. Influência da exposição gengival na estética do sorriso. Dental Press J Orthod 2011; $16: 5111$-18.

3. E SILVA NETO JMDEA, et al. Protocolos de Aplicação de Toxina para Sorriso Gengival: uma revisão de literatura. Revista Eletrônica Acervo Saúde, n. 28, p. e1079, 13 ago. 2019.

4. FLÁVIO A. Toxina Botulínica para harmonização facial. 1 ed. São Paulo: Napoleão Ltda; 2019; 78-95.

5. KANE M, SATTLER G. Guia ilustrado para infiltrações estéticas com toxina botulínica: base, localização, utilidades. São Paulo: Di Livros, 2016.

6. KEANEY TC, ALSTER TS. Botulinum toxin in men: review of relevant anatomy and clinical trial data. World Environment Day 2013; 39(10):1434-43.

7. KLEIN AW. Complications and adverse reactionswith the use ofbotulinumtoxin. Dis Mon, v. 48, n. 5, 2002; p. 336356.

8. MACEDO ACVB, et al. O sorriso gengival-tratamento baseado na etiologia: uma revisão de literatura. Revista Periodontia, 2012; 22(4): 36-44.

9. MADEIRA MC. Anatomia da face: bades anátomo-funcionais para a prática odontológica. 3 ed. São Paulo: SARVIER, 2001; 67-96

10. MAGRO AKD. Tratamento do sorriso gengival com toxina botulínica tipo A: relato de caso. RFO, Passo Fundo, 2015; 20(1): 81-87, jan./abr. 
11. MATOS MB, et al. O uso da toxina botulínica na correção do sorriso gengival - revisão de literatura. Braz $\mathrm{J}$ Periodontol, 2017; 27: 29-36.

12. MOCK D, et al. Botulinum Toxin and Dentistry. In: Ensuring Continued Trust. Royal College of Dental Surgeons of Ontario, Toronto, 2009; 1-4.

13. OKAJIMA M. Toxina botulínica: da terapia a estética. Ident, 2013.

14. OLIVEIRA MT, MOLINA RO. Sorriso Gengival, quando a toxina botulínica pode ser utilizada. Rev. Odontol. Araçatuba, 2011; $32(2):$ p.58-61.

15. PANOSSIAN AJ, BLOCK MS. Evaluation of the Smile: Facial and Dental Considerations. J Oral Maxillofac Surg 2010; 68: $547-554$

16. PEDRON IG. Aplicação da toxina botulínica associada à clínica integrada no tratamento do sorriso gengival. Revista do Instituto de Ciências da Saúde, São Paulo, v. 32, n. 4, 2014; p. 365-369.

17. PEDRON IG. Cuidados no planejamento para a aplicação da Toxina Botulínica em Sorriso Gengival. Revista Odontol. São Paulo: UNICID; 2014; 26 (3): 250-6.

18. SENISE IR, et al. O uso de toxina botulínica como alternativa para o tratamento do sorriso gengival causado pela hiperatividade do lábio superior. Maringá: Revista UNINGÁ Review, 2015; 23(3): 104-110.

19. SEVILHA FM, et al. Toxina botulínica tipo a: uma alternativa para tratamentos odontológicos. Revista Sobrape, São Paulo, v. 21, n. 2, 2011; p. 12-17.

20. SPOSITO MMM, TEIXEIRA SAF. Toxina botulínica tipo a para bruxismo: análise sistemática. Acta Fisiátrica, São Paulo, v. 21, n. 4, 2014; p. 201-204.

21. SPOSITO MMM. Toxina botulínica tipo A - propriedades farmacológicas e uso clínico. Acta Fisiátrica, $2004 ; 1: 7-44$. 